\title{
218. On a Theorem of Bouldin
}

\author{
By Ritsuo NaKamoto \\ Tennoji Senior Highschool \\ (Comm. by Kinjirô KunUGi, M. J. A., Oct. 12, 1971)
}

1. Introduction. In this paper $A$ means a bounded linear operator on a complex Hilbert space $\mathfrak{S}$ and the numerical range $W(A)$ means the set of complex numbers

$$
W(A)=\{(A x \mid x) ; x \in \mathfrak{S},\|x\|=1\} .
$$

It is well known that $W(A)$ is convex and the closure $\overline{W(A)}$ of $W(A)$ contains the spectrum $\sigma(A)$ of $A$.

Recently, R. Bouldin [2] [3] has shown an elegant result which determines the numerical range of the product $A B$ in terms of $\overline{W(A)}$ and $\overline{W(B)}$ under a suitable condition (cf. Theorem 2 in the below).

The purpose of the present paper is to give an elementary proof of Bouldin's theorem.

The author expresses his cordial thanks to Prof. T. Furuta to whom the author is largely indebted.

2. Bouldin's theorem. We shall show at first the following theorem by a simple calculation:

Theorem 1. If $A$ is an operator and $B$ is nonnegative then $W\left(B^{1 / 2} A B^{1 / 2}\right) \subset W(A) W(B)$.

Proof. If $B^{1 / 2} x \neq 0$ for a unit vector, then

$$
\begin{aligned}
\left(B^{1 / 2} A B^{1 / 2} x \mid x\right) & =\left(A B^{1 / 2} x \mid B^{1 / 2} x\right) \\
& =\left\|B^{1 / 2} x\right\|^{2}\left(A \frac{B^{1 / 2} x}{\left\|B^{1 / 2} x\right\|} \mid \frac{B^{1 / 2} x}{\left\|B^{1 / 2} x\right\|}\right) \\
& =(B x \mid x)\left(A \frac{B^{1 / 2} x}{\left\|B^{1 / 2} x\right\|} \mid \frac{B^{1 / 2} x}{\left\|B^{1 / 2} x\right\|}\right) .
\end{aligned}
$$

If $B^{1 / 2} x=0$, then

$$
\left(B^{1 / 2} A B^{1 / 2} x \mid x\right)=0=(B x \mid x) .
$$

Therefore the theorem is proved.

Remark. Since

Theorem 1 implies at once

$$
\sigma(A B)=\sigma\left(B^{1 / 2} A B^{1 / 2}\right) \pm\{0\},
$$

$$
\sigma(A B) \subset \overline{W(A}) \overline{W(B)}
$$

under the same conditions of Theorem 1.

The above considerations imply at once the following theorem which is originally obtained by a help of perturbation theory:

Theorem 2 (Bouldin). If $A$ is an operator and $B$ is nonnegative then 


$$
\operatorname{co} \sigma(A B) \subset \overline{W(A)} \overline{W(B)},
$$

where co $X$ means the convex hull of $X$.

3. Applications. The materials of this section are based on an idea due to Prof. T. Furuta.

S. K. Berberian [1] introduced the following notion: A unitary operator is cramped if its spectrum is contained in an open semi-circle of the unit circle. A closed sector $S$ is cramped if

$$
S=\left\{r e^{i \theta} ; r>0, \theta_{1} \leqq \theta \leqq \theta_{2}, \theta_{2}-\theta_{1}<\pi\right\}
$$

$S(X)$ denotes the least (closed) sector containing $X$. The boundary of a sector, which consists of two half lines, will be called the sector lines.

An importance of Bouldin's theorem lies in the fact that one can locate the spectrum $\sigma(T)$ of $T$ in the sector determined by $W(U)$ using the polar decomposition $T=U H$.

According to a definition of Halmos [5], an operator $A$ is said to be convexoid if

$$
\operatorname{co} \sigma(A)=\overline{W(A})
$$

Theorem 3. If $A$ and $A B$ are convexoids such that $0 \notin \overline{W(A)}$ and $B$ is an invertible nonnegative operator, then

(i) $0 \notin \overline{W(A B)}$, and

(ii) $\overline{W(A B})$ and $\overline{W(A)} \overline{W(B})$ have the same cramped sector.

Proof. By Bouldin's theorem, the hypothesis implies

$$
0 \notin \operatorname{co} \sigma(A B)=\overline{W(A B)} \subset \overline{W(A}) \overline{W(B}) \text {, }
$$

which implies

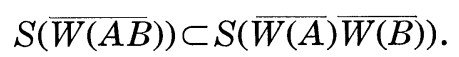

The reverse inclusion follows from the relation

$$
\left.\left.0 \notin \overline{W(A})=\operatorname{co} \sigma(A)=\operatorname{co} \sigma\left(A B B^{-1}\right) \subset \overline{W(A B}\right) \overline{W\left(B^{-1}\right.}\right)
$$

since

$$
\overline{W(B)}, \overline{W\left(B^{-1}\right)} \subset\langle 0, \infty\rangle .
$$

By Theorems 1 and 3, we have the following corollaries:

Corollary 4. If $B$ is nonnegative and invertible, $A$ is convexoid such that $0 \notin \overline{W(A)}$ and $A B=B A$ then (i) and (ii) of Theorem 2 hold.

Corollary 5. If $A$ is convexoid and $B$ is an invertible nonnegative operator. Moreover, $A B$ is convexoid, then the following conditions are equivalent:

(i) $0 \notin \overline{W(A)}$, and

(ii) $0 \notin \overline{W(A B)}$.

Let $T=U H$ be the polar decomposition of $T$. If $T$ is invertible, then $H$ is strictly positive and $U$ is unitary so that we have the following corollaries by Theorems 2 and 3 :

Corollary 6 ([4]). If $T=U H$ is an invertible convexoid such that $U$ is cramped, then

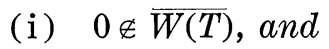


(ii) $\overline{W(T)}$ and $\overline{W(U)}$ have the same cramped sector.

Corollary 7 (Berberian in [6]). Let $T$ be an invertible operator such that $U=T\left(T^{*} T\right)^{-1 / 2}$ is cramped, then $0 \notin \operatorname{co} \sigma(T)$.

\section{References}

[1] S. K. Berberian: The numerical range of a normal operator. Duke Math. J., 31, 479-483 (1964).

[2] R. Bouldin: The numerical range of a product. J. Math. Anal. Appl., 32, 459-467 (1970).

[ 3 ] _ : The numerical range of a product. II. J. Math. Anal. Appl., 33, 212-219 (1971).

[ 4 ] T. Furuta and R. Nakamoto: On some theorems of Berberian and Sheth. Proc. Japan Acad., 46, 841-845 (1970).

[5] P. R. Halmos: A Hilbert Space Problem Book. Van Nostrand, Princeton (1967).

[6] V. Istrăţescu: On some classes of operators. Math. Ann., 188, 227-232 (1970). 